the first-line maintenance setting via Early Access Programmes (Italy, UK), Temporary Use Authorization (France) or reimbursement following regulatory approval (Italy, UK). Main study endpoint is real-world PFS. Secondary endpoints include overall survival and response rates. The study will also describe surrogate measures of response and tolerability, including time to discontinuation, dose modifications (with reasons) and time to first and second subsequent treatment. Outcomes will be described by key subgroup status pre-index, including performance status, FIGO stage, BRCAm status, debulking surgery outcome and clinical response to chemotherapy. The study aims to include 350 patients. Retrospective data collection began in December 2020 and is planned to end by Q3 2023. As of April 2021, 69 patients have participated.

\section{UPLIFT (ENGOT-OV67/GOG-3048) A PIVOTAL COHORT OF UPIFITAMAB RILSODOTIN, A NAPI2B-DIRECTED ADC IN PLATINUM-RESISTANT OVARIAN CANCER}

${ }^{1} \mathrm{~N}$ Concin*, ${ }^{2,3}{ }^{\mathrm{E}}$ Hamilton, ${ }^{4} \mathrm{LM}$ Randall, ${ }^{5} \mathrm{~S}$ Banerjee, ${ }^{6} \mathrm{~L}$ Mileshkin, ${ }^{7,8} \mathrm{RL}$ Coleman, 9,10,11 B Monk, ${ }^{12,13,14,15,16,17}$ MR Mirza, ${ }^{18} \mathrm{~A}$ Savarese, ${ }^{19}$ I Ray-Coquard, ${ }^{20} \mathrm{JA}$ Perez Fidalgo, ${ }^{21} \mathrm{P}$ Bernardo, ${ }^{21} \mathrm{E}$ Putiri, ${ }^{21} \mathrm{R}$ Burger, ${ }^{21} \mathrm{R}$ Mosher, ${ }^{22} \mathrm{D}$ Richardson, ${ }^{23} \mathrm{D}$ Cibula, ${ }^{24} \mathrm{R}$ Madry, ${ }^{25} \mathrm{R}$ Chekerov, ${ }^{26} \mathrm{~T}$ Van Gorp. 'Medical University of Innsbruck, Austria, Department of Obstetrics and Gynaecology, Innsbruck, Austria; ${ }^{2}$ Sarah Cannon Research Institute, Nashville, United States; ${ }^{3}$ Tennessee Oncology, Nashville, United States; ${ }^{4}$ VCU Health System, MCV Physicians, Department of Obstetrics and Gynecology Oncology, Richmond, United States; ${ }^{5}$ The Royal Marsden Nhs Trust, Institute of Cancer Research, London, United Kingdom; ${ }^{6}$ Peter MacCallum Cancer Centre, Melbourne, Australia; ${ }^{7}$ Texas Oncology-The Woodlands, The Woodlands, United States; ${ }^{8}$ GOG Foundation; ${ }^{9}$ Arizona Oncology, US Oncology Network, Phoenix, United States; ${ }^{10}$ US Oncology, Gynecologic Program; ${ }^{11}$ GOG Partners; ${ }^{12}$ Rigshospitalet, København, Denmark; ${ }^{13}$ Nordic Society of Gynecological Oncology, Clinical Trial Unit, Denmark; ${ }^{14}$ European Network of Gynecological Trial groups; ${ }^{15}$ Gynecologic Cancer Inter-Group; ${ }^{16}$ European Society of Medical Oncology; ${ }^{17}$ European Society of Gynecological Oncology; ${ }^{18}$ Regina Elena National Cancer Institute, Rome, Italy; ${ }^{19}$ Léon Bérard Center, Lyon, France; ${ }^{20}$ Hospital Clínic Universitari, València, Spain; ${ }^{21}$ Mersana Therapeutics Inc, Cambridge, United States; ${ }^{22} \mathrm{OU}$ Health Stephenson Cancer Center, Oklahoma City, United States; ${ }^{23}$ Charles University, Czech Republic; ${ }^{24}$ Poznan University of Medical Sciences, Poznań, Poland; ${ }^{25}$ Charité - Universitätsmedizin Berlin, Berlin, Germany; ${ }^{26} \mathrm{Uz}$ Leuven, oncology, Leuven, Belgium

\subsection{6/ijgc-2021-ESGO.351}

Introduction/Background* Upifitamab rilsodotin (XMT-1536; UpRi), is a first-in-class Dolaflexin antibody-drug conjugate targeting NaPi2b, a sodium-dependent phosphate transport protein broadly expressed in solid tumors including high-grade serous epithelial ovarian cancer (OC). UpRi's safety and efficacy are being evaluated in a Phase I study (NCT03319628). Preliminary antitumor activity from an expansion cohort of heavily-pretreated OC patients has been reported (Hamilton et al, ESMO 2020). A data-cut of December 2020 demonstrated an ORR of $39 \%$ including 2 CRs and DCR of $81 \%$ in 26 OC patients with high NaPi2b expression (TPS $\geq 75$ ). The 2 patients achieving CR had previously been treated with bevacizumab and PARPi (Richardson et al, ASCO 2021, TPS5607). The prevalence of a TPS $\geq 75$ is greater than $60 \%$.

PROC remains a serious unmet medical need as available treatment options provide modest benefit of no more than $12 \%$ ORR and median OS less than 12 months. Based on encouraging anti-tumor activity of UpRi, UPLIFT was designed as a Phase 2 single-arm registration strategy for PROC as part of the ongoing study.

Methodology The UPLIFT cohort is enrolling patients with platinum resistant high grade serous ovarian, fallopian tube and primary peritoneal cancer with up to 4 prior lines of therapy. Prior bevacizumab is required for patients with 1 or 2 prior lines of therapy but is not required for patients with 3-4 prior lines of therapy. UPLIFT will enroll approximately 180 patients globally for 100 patients with high $\mathrm{NaPi} 2 \mathrm{~b}$ expression. UpRi is dosed intravenously at $43 \mathrm{mg} / \mathrm{m} 2$ every 4 weeks. Patients may enroll regardless of $\mathrm{NaPi} 2 \mathrm{~b}$ expression and regardless of baseline peripheral neuropathy. Baseline tumor samples (fresh or archived) will be collected for retrospective tumor tissue evaluation of $\mathrm{NaPi} 2 \mathrm{~b}$ expression.

Result(s)* The primary objective is assessment of objective response rate in patients with high $\mathrm{NaPi} 2 \mathrm{~b}$ expression. The cut-off for high $\mathrm{NaPi} 2 \mathrm{~b}$ expression is TPS $\geq 75$ and was based on data from the expansion cohort. Secondary endpoints include objective response rate in the overall population, duration of response, and adverse events.

Conclusion* This study is being conducted in collaboration with ENGOT and GOG. Patients will be enrolled globally. (NCT03319628).

\section{SURGICAL OUTCOME AS PROGNOSTIC FACTOR IN DIFFERENT HISTOLOGIC SUBTYPES OF OVARIAN CARCINOMA- ANALYSIS OF 7 PHASE III TRIALS BY AGO STUDYGROUP + ENGOT}

${ }^{1,2} \mathrm{~F} \mathrm{Heitz}^{*},{ }^{1,2} \mathrm{~A}$ Du Bois, ${ }^{2,3} \mathrm{~A}$ Reuß, ${ }^{4} \mathrm{E}$ Pujade-Lauraine, ${ }^{5} \mathrm{MR}$ Mirza, ${ }^{6} \mathrm{~S}$ Greggi, ${ }^{7} \mathrm{~N}$ Colombo, ${ }^{8} \mathrm{C}$ Marth, ${ }^{9} \mathrm{~B}$ Vergote, ${ }^{1,2} \mathrm{P}$ Harter, ${ }^{10}$ Ray-Coquard, ${ }^{11} \mathrm{~GB}$ Kristensen, ${ }^{2,12} \mathrm{~S}$ Mahner, ${ }^{13} \mathrm{AC}$ Hardy, ${ }^{14} \mathrm{~J}$ Sehouli, ${ }^{2,15} \mathrm{p}$ Wimberger, ${ }^{2,16} \mathrm{M}$ Eichbaum, ${ }^{2} \mathrm{G}$ Elser ${ }^{2,17} \mathrm{~W}$ Meier, ${ }^{2,18} \mathrm{~J}$ Pfisterer. ${ }^{1}$ Kliniken Essen-Mitte, Gynecology and Gynecologic Oncology; ${ }^{2}$ AGO Study Group, Wiesbaden; ${ }^{3}$ Philipps University Marburg, Coordinating Center for Clinical Trials of the Philipps-University of Marburg, Marburg, Germany; ${ }^{4}$ GINECO and Hôpital Dieu, Paris, France; ${ }^{5}$ NSGO \& Rigshospitalet, Dept. of Oncology; Copenhagen University Hospital," Copenhagen; ${ }^{6}$ MITO \& Istituto Nazionale per lo Studio e la Cura dei Tumori di Napoli, Gynecologic Oncology, Naples, Italy; ${ }^{7} \mathrm{MaNGO}$ \& European Institute of Oncology, Gynecologic Oncology, Milano, Italy; ${ }^{8}$ AGO-A \& Innsbruck Medical University, Department of Obstetrics and Gynecology, Innsbruck; ${ }^{9} B G O G$ \& University Hospitals Leuven, Dept. of Gynaecological Oncology, Leuven, Belgium; ${ }^{10}$ GINECO \& Centre Leon Bérard Université Claude Bernard Lyon Est, , Lyon, France; ${ }^{11}$ NSGO \& Oslo University Hospital, Oslo, Norway; ${ }^{12}$ University Hospital, LMU Munich, Department of Obstetrics and Gynecology; ${ }^{13}$ GINECO \& Hôpital Privé des Côtes d'Armor, Plérin; ${ }^{14}$ NOGGO \& Charité Universitätsmedizin Berlin, Campus Virchow, Dpt. Gynecology with Center for Oncological Surgery, Berlin, Germany; ${ }^{15}$ University Hospital Essen and University Hospital Carl Gustav Carus, TU Dresden, Department of Gynecology and Obstetrics, Dresden; ${ }^{16}$ Helios Dr. Horst Schmidt Kliniken Wiesbaden, Department of Gynecology, Wiesbaden; ${ }^{17}$ Düsseldorf University Hospital and Evangelisches Krankenhaus Düsseldorf, Department of Gynecology and Obstetrics, Düsseldorf, Germany; ${ }^{8}$ Gynecologic Oncology Center, Kiel, Germany

\subsection{6/ijgc-2021-ESGO.352}

Introduction/Background" Debulking surgery is the mainstay of treatment for patients (pts) with advanced epithelial ovarian cancer (EOC). Upfront surgery (PDS) with complete macroscopic resection (TR0) is associated with best survival while PDS to small residual disease (TR1-10) provides moderate benefit in high grade serous OC. The impact of resection status in other histological subtypes so far has not been defined and especially the role of TR1-10 is under debate. This analysis should help to better understand the interplay between histological subtype, surgical outcome, and prognosis.

Methodology Data of patients (>FIGO IIIB) from 7 AGOStudygroup led phase III multicentre trials (AGO-OVAR 3,5,7,9,11,12,15), (1995-2011) were pooled and analysed with focus on PDS resection status on overall survival (OS) in different histological subtypes: low grade (low grade serous or endometrioid), mucinous, clear cell, and high-grade (e.g. 

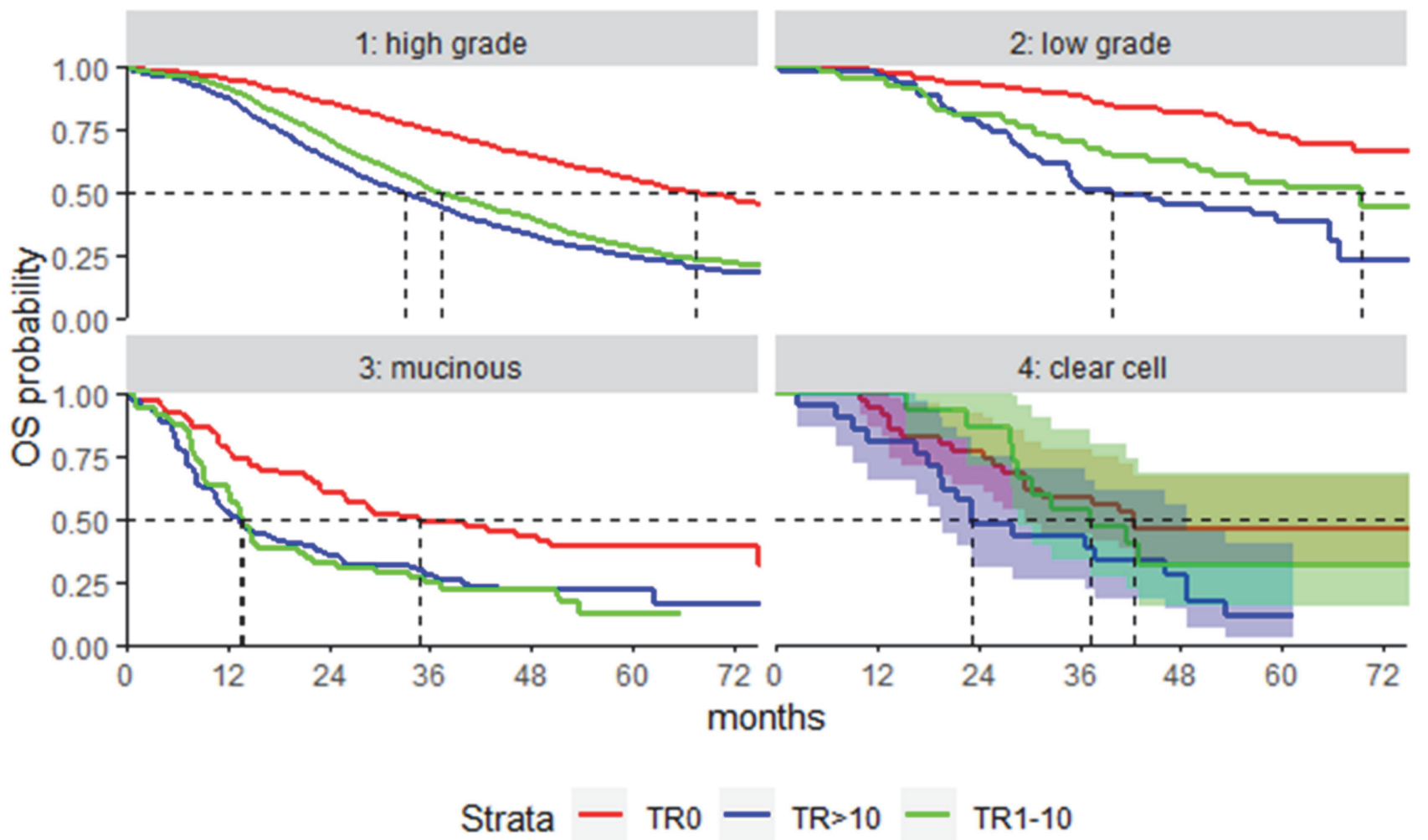

Abstract 186 Figure 1 Kaplan-Meier plots of overall survival within tumour types according to complete (TR0) and largely (TR1-10mm) debulking; number of patients included into the clear cell cohort was too small to allow meaningful OS analyses

\begin{tabular}{|c|c|c|c|c|}
\hline & High grade & Low grade & Mucinous & Clear cell \\
\hline $\mathrm{n}$ & 5,156 & 299 & 219 & 71 \\
\hline TRO (\%) & $32.7 \%$ & $52.2 \%$ & $31.1 \%$ & $49.3 \%$ \\
\hline TR1-10 (\%) & $31.7 \%$ & $26.1 \%$ & $26.0 \%$ & $21.1 \%$ \\
\hline OR $(95 \% \mathrm{Cl})$ for TRO & 1 (reference) & $\begin{array}{l}1.84(1.44- \\
2.36)\end{array}$ & $\begin{array}{l}0.95(0.70- \\
1.29)\end{array}$ & $\begin{array}{l}1.69(1.03- \\
2.79)\end{array}$ \\
\hline Deaths n (\%) & $\begin{array}{l}3,122 \\
(60.6 \%)\end{array}$ & $115(38.5 \%)$ & $155(70.8 \%)$ & $46(64.8 \%)$ \\
\hline $\begin{array}{l}\text { OS HR(95\%Cl) TRO vs } \\
\text { TR>10 }\end{array}$ & $\begin{array}{l}0.48(0.43- \\
0.52)\end{array}$ & $\begin{array}{l}0.39(0.24- \\
0.64)\end{array}$ & $\begin{array}{l}0.64(0.40- \\
1.03)\end{array}$ & * \\
\hline $\begin{array}{l}\text { OS HR(95\%Cl) TR1-10 vs } \\
\text { TR>10 }\end{array}$ & $\begin{array}{l}0.91(0.84- \\
0.99)\end{array}$ & $\begin{array}{l}0.87(0.52- \\
1.44)\end{array}$ & $\begin{array}{l}1.09(0.71- \\
1.67)\end{array}$ & * \\
\hline $\begin{array}{l}\text { OS HR(95\%Cl) TRO vs } \\
\text { TR1-10 }\end{array}$ & $\begin{array}{l}0.52(0.48- \\
0.58)\end{array}$ & $\begin{array}{l}0.45(0.28- \\
0.73)\end{array}$ & $\begin{array}{l}0.59(0.36- \\
0.96)\end{array}$ & * \\
\hline $\begin{array}{l}\text { OS HR(95\%Cl) TRO vs } \\
\text { TR>0 }\end{array}$ & $\begin{array}{l}0.50(0.46- \\
0.54)\end{array}$ & $\begin{array}{l}0.42(0.28- \\
0.64)\end{array}$ & $\begin{array}{l}0.62(0.40- \\
0.95)\end{array}$ & * \\
\hline
\end{tabular}

serous, endometrioid, undifferentiated histology). Multiple logistic regression of achieving TR0 in the full population and multiple Cox-regression of OS separately in each histological subpopulation adjusting potential confounders (treatment arm within each study, ECOG performance status, age, FIGO stage) were undertaken.

Result(s)* 5,745 pts were eligible (5,156 high grade, 299 low grade, 219 mucinous, 71 clear cell). Differences in resection rates between histological subtypes are displayed in the table
1. Adjusted odds ratios showed significantly higher odds for achieving TR0 for low grade and clear cell compared to highgrade, (table 1). Median follow-up was 60.7 months. The figure shows OS within tumour types according to resection status. Hazard ratios between TR0, TR1-10 and TR $>10$ obtained from multiple Cox regression of OS are shown in the table 1 .

Conclusion* Our analysis confirmed the value of complete resection in all subtypes of ovarian cancer. The role of largely resection (TR1-10mm) seemed to be relevant in low grade OC, but marginal although significant in HGSOC and inconclusive in mucinous and clear cell OC. Larger databases are necessary to gain more reliable data in these subgroups.

\section{PLATINUM RESISTANCE IN OVARIAN CARCINOMA LONG-TERM SURVIVORS: A RETROSPECTIVE STUDY AT THE TUEBINGEN UNIVERSITY WOMEN'S HOSPITAL}

${ }^{1} S$ Mittelstadt*, 'M Grube, ${ }^{1} T$ Engler, ${ }^{1,2} E$ Oberlechner, ${ }^{1} S$ Wörz, ${ }^{1} S$ Matovina, ${ }^{1} A$ Stöhr, ${ }^{1} \mathrm{~L}$ Volmer, ${ }^{1} \mathrm{EM}$ Grischke, ${ }^{1} \mathrm{CB}$ Walter, ${ }^{1} \mathrm{~A}$ Hartkopf, ${ }^{3} \mathrm{~S}$ Kommoss. ${ }^{1}$ Tuebingen University Hospital Department of Women's Health; ${ }^{2}$ University Hospital Tuebingen, Department of Women's Health, Tuebingen, Germany; ${ }^{3}$ Tuebingen University Hospital, Department of Women's Health, Tuebingen, Germany

\subsection{6/ijgc-2021-ESGO.353}

Introduction/Background* Despite the majority of ovarian carcinoma patients $(\mathrm{OvCa})$ initially responding to primary therapy, relapse occurs frequently in early and advanced stages (25 and $80 \%$ respectively) and $\mathrm{OvCa}$ is still associated with an overall poor prognosis. Nevertheless, therapy response and disease progression can be quite variable and long-term survival is reported in up to $30 \%$ of patients. It is known that, 